\title{
Prompt Rise in Urinary Ammonium Excretion Suffices To Mitigate Metabolic Acidosis in an Experimental Animal Model of Severe Normovolemic Hemodilution
}

\author{
J. K. TELOH ${ }^{1}$, I. N. WAACK ${ }^{\mathbf{1}},{ }^{\dagger}$ H. DE GROOT $^{\mathbf{1}}$ \\ ${ }^{\dagger}$ Deceased May 10, 2016. \\ ${ }^{1}$ Institute of Physiological Chemistry, University of Duisburg-Essen, University Hospital Essen, \\ Essen, Germany
}

Received September 21, 2016

Accepted January 25, 2017

On-line April 12, 2017

\section{Summary}

Recently, we have established a model of severe stepwise normovolemic hemodilution to a hematocrit of $10 \%$ in rats employing three different colloidal volume replacement solutions (Voluven, Volulyte and Gelafundin) that are routinely used in clinical practice at present. We did not see severe dilutional acidosis as to be expected, but a decline in urinary $\mathrm{pH}$. We here looked on further mechanisms of renal acid excretion during normovolemic hemodilution. Bicarbonate, which had been removed during normovolemic hemodilution, was calculated with the help of the Henderson-Hasselbalch equation. The urinary amount of ammonium as well as phosphate was determined in residual probes. The absolute amount of free protons in urine was obtained from the $\mathrm{pH}$ of the respective samples. The amount of protons generated during normovolemic hemodilution was approximately $0.6 \mathrm{mmol}$. During experimental time $(5.5 \mathrm{~h})$, distinct urinary ammonium excretion occurred (Voluven $0.52 \mathrm{mmol}$, Volulyte $0.39 \mathrm{mmol}$ and Gelafundin $0.77 \mathrm{mmol}$ ). Proton excretion via the phosphate buffer constituted $0.04 \mathrm{mmol}$ in every experimental group. Excretion of free protons was in the range of $10^{-6} \mathrm{mmol}$. The present data prove that the prompt rise in urinary ammonium excretion is also valid for acute metabolic acidosis originating from severe normovolemic hemodilution.

\section{Key words}

Acute metabolic acidosis - Ammonium excretion - Acid-base homeostasis • Normovolemic hemodilution • Renal acid excretion

\section{Corresponding author}

J. K. Teloh, Institute of Physiological Chemistry, University of Duisburg-Essen, University Hospital Essen, Hufelandstraße 55, 45122 Essen, Germany. Fax: +(49) 201-723 5943. E-mail: Johanna.teloh@uk-essen.de

\section{Introduction}

Metabolic acidosis is defined as a condition in which plasma bicarbonate is reduced to a degree that equals a base excess below $-2 \mathrm{mEq} / 1$ (Astrup et al. 1960, Kraut and Madias 2012). Severe manifestation of this type of acid-base disturbance is associated with increased morbidity and mortality, and therefore with poor clinical outcome (Gunnerson et al. 2006, Kraut and Madias 2012). The miserable prognosis can be explained by the fact that acute metabolic acidosis is known to have several deleterious effects, a decrease in cardiac contractility and cardiac output, hypotension due to peripheral vasodilation, and decreased binding of insulin and catecholamines, for instance (Oliva 1970). In Western countries, most cases of acidosis with metabolic rather than respiratory origin are caused by lactic acidosis or ketoacidosis (Kraut and Madias 2012). Nonetheless, in clinical situations that make the administration of large volumes of saline fluids necessary, a metabolic acidosis might occur due to dilutional effects. Dilutional acidosis by definition develops, if the buffer base bicarbonate is diluted due to administration of volume replacement solutions that are devoid of bicarbonate, whereas the 
buffer acid carbon dioxide is not (Waters et al. 1999). In the clinical setting outlined above, colloidal volume replacement solutions are often used due to their oncotic properties in contrast to pure crystalloid solutions. Concerning their composition, colloidal solutions differ as regards the contained colloid, electrolytes and possibly metabolizable anions.

Recently, we have established a model of severe stepwise normovolemic hemodilution to a final hematocrit of $10 \pm 1 \%$ in rats (Teloh et al. 2013). For hemodilution, we employed three different approved volume replacement solutions (one per experimental group), which all have been commonly used in clinical routine (Gelafundin, Voluven, Volulyte). Starting at a value of $-0.6 \mathrm{mEq} / \mathrm{l}$, current base excess fell to a value of $-3.5 \mathrm{mEq} / \mathrm{l}$ at the end of the experiment in all groups, indeed indicating the existence of an acute metabolic acidosis, but to a much lesser extent than expected. Despite the severe degree of hemodilution, we did not detect any drop in plasma $\mathrm{pH}$ in none of the three experimental groups (Gelafundin, Voluven, Volulyte), instead staying at a rather constant level. To find an explanation for this unexpected finding and since the urinary $\mathrm{pH}$ decreased in all groups from an initial value slightly above neutrality to approximately 6.3 in the observation phase, we decided to measure phosphate and ammonium content in residual urine probes of this experiment and compared these values quantitatively with the amount of bicarbonate removed during stepwise severe normovolemic hemodilution.

\section{Methods}

We took residual urine probes from our previous experiments (Teloh et al. 2013). In those experiments, stepwise normovolemic hemodilution in male Wistar rats (strain WIS/WU, body weight 410-470 g, estimated blood volume of $25-29 \mathrm{ml}$ according to the equation of Lee and Blaufox 1985) until a final hematocrit of $10 \pm 1 \%$ using one of the colloidal volume replacement solutions Voluven, Volulyte or Gelafundin was performed. For blood withdrawal and volume replacement, one catheter was placed within the right femoral artery and the right femoral vein each. Per step of dilution, $3 \mathrm{ml}$ of blood were exchanged, followed by a pause interval of $15 \mathrm{~min}$. For urine collection, a catheter was inserted into the bladder and the urine collected hereby pooled for the respective phase of the experiment (initial, phase of dilution and observation phase).

\section{Measurements}

For ammonium quantification, capillary electrophoresis was used. For this purpose, a fused silica capillary was employed with an effective length of $50 \mathrm{~cm}$, an I.D. of $75 \mu \mathrm{m}$ and an O.D. of $375 \mu \mathrm{m}$. Samples of initial urine were diluted with ultrapure water 1:50, whereas samples of the phase of dilution as well as observation phase were diluted 1:100. Analysis was performed using a cation analysis kit (ABSciex, Fullerton, USA). Per analysis, the volume was applied using pressure injection. The subsequent separation proceeded using a voltage of $30 \mathrm{kV}$ and normal polarity of the capillary. Indirect detection was performed employing a photo diode array at a wavelength of $200 \mathrm{~nm}$.

Quantification of phosphate in urine samples was realized with the help of a colorimetric test at a wavelength of $340 \mathrm{~nm}$ (Daly and Ertingshausen 1972). Here, inorganic phosphate reacts with ammonium molybdate to form an unreduced phosphomolybdate complex. The obtained results had already been corrected for background absorption by individual blank measurements that were automatically subtracted from the sample's absorbance by the analyzer.

\section{Calculations}

Bicarbonate having been removed during every single step of normovolemic hemodilution was calculated with the help of the Henderson-Hasselbalch equation on the basis of measured $\mathrm{pH}$ and carbon dioxide partial pressure at the respective point of time (Teloh et al. 2013). It was assumed, that during the pause interval with a length of $15 \mathrm{~min}$ after every step of dilution, a new equilibrium had adjusted.

The absolute amount of free protons in urine, which is responsible for its acidification, was obtained from the $\mathrm{pH}$ of the respective sample (Teloh et al. 2013). Absolute amounts of ammonium as well as phosphate were calculated taking into account the volumes of the respective urine samples.

\section{Statistical analysis}

Experiments were performed with six animals per group. The data are expressed as mean values \pm standard error of the mean (SEM). Comparisons within a single group were performed using repeated measurement two-way independent analysis of variance (ANOVA) followed by a Dunnett post hoc analysis. Comparisons among multiple groups were performed 
during two-way independent ANOVA followed by a Tukey post hoc analysis. A $P$ value $<0.05$ was considered significant.

\section{Results}

The absolute amount of ammonium in initial urine varied between $0.012 \pm 0.003 \mathrm{mmol}$ (Volulyte group) and $0.021 \pm 0.005 \mathrm{mmol}$ (Gelafundin group) (Fig. 1). During the phase of dilution, the absolute amount of ammonium increased to values of $0.194 \pm 0.022 \mathrm{mmol}, 0.296 \pm 0.024 \mathrm{mmol}$ for the Volulyte

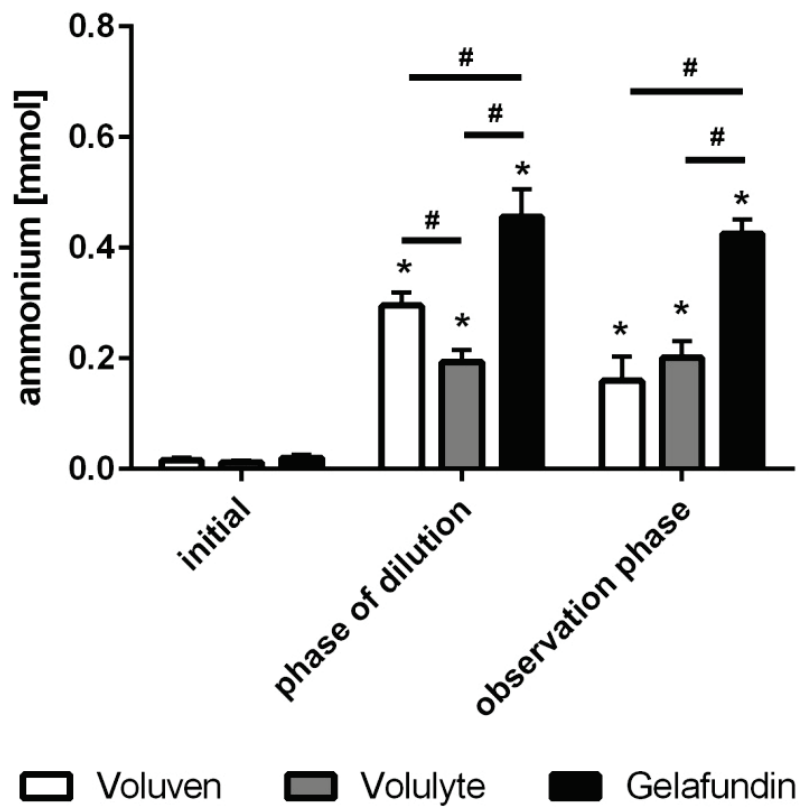

Fig. 1. Ammonia excretion during and subsequent to severe normovolemic hemodilution with Voluven, Volulyte and Gelafundin. After insertion of the urinary catheter, the initial urine was collected. Collection was continued during the phase of hemodilution as well as during the observation phase. Experimental groups differ from each other in the colloidal volume replacement solution employed for normovolemic hemodilution. * $p<0.05$ compared with the initial value. \# $p<0.05$. Sample size for every group: $n=3$ at initial due to the small urine volume; $n=6$ at phase of dilution; $\mathrm{n}=4$ at observation phase due to 2 missing samples. and Voluven group, but to $0.455 \pm 0.051 \mathrm{mmol}$ for the Gelafundin group. In the observation phase, the values amounted to $0.201 \pm 0.030 \mathrm{mmol}, 0.160 \pm 0.044 \mathrm{mmol}$ and $0.425 \pm 0.026 \mathrm{mmol}$, respectively.

The absolute phosphate amount in initial urine was $0.0004 \pm 0.0002 \mathrm{mmol}$ (Voluven group) and $0.0010 \pm 0.0004 \mathrm{mmol}$ (Gelafundin group) (Fig. 2). The urinary amount increased to $0.038 \pm 0.002 \mathrm{mmol}$, $0.015 \pm 0.007 \mathrm{mmol}$ and $0.032 \pm 0.012 \mathrm{mmol}$ (Voluven, Volulyte, Gelafundin) in the phase of dilution and was $0.012 \pm 0.003 \mathrm{mmol}, 0.022 \pm 0.014 \mathrm{mmol}$ and $0.011 \pm 0.005$ mmol, respectively, in the observation phase.

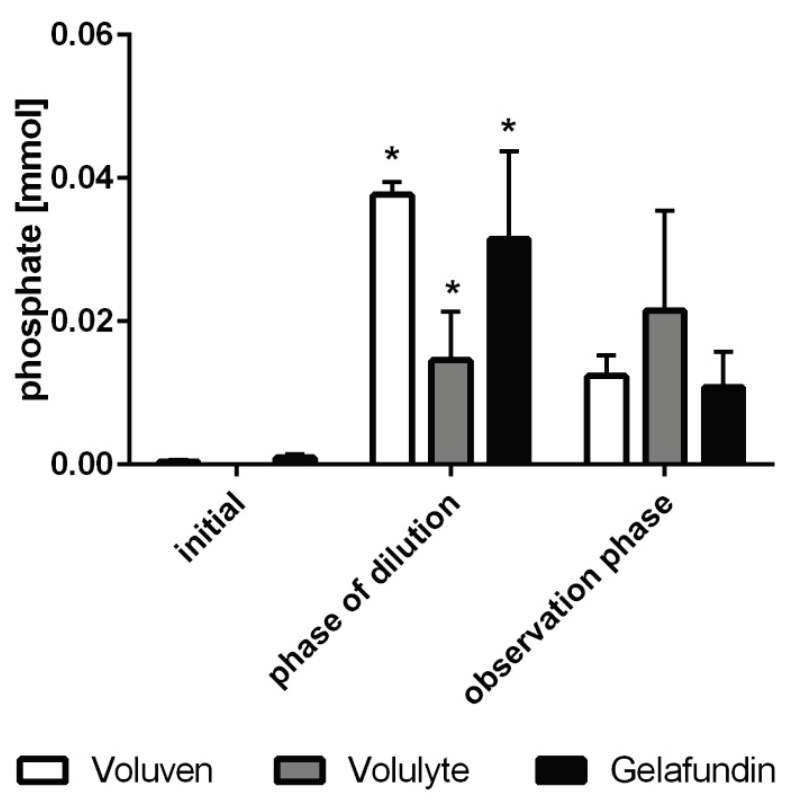

Fig. 2. Phosphate excretion during and subsequent to severe normovolemic hemodilution with Voluven, Volulyte and Gelafundin. After insertion of the urinary catheter, the initial urine was collected. Collection was continued during the phase of hemodilution as well as during the observation phase. Experimental groups differ from each other in the colloidal volume replacement solution employed for normovolemic hemodilution. * $* 0.05$ compared with the initial value. Sample size for every group: $n=3$ at initial due to the small urine volume; $n=6$ at phase of dilution; $\mathrm{n}=4$ at observation phase due to 2 missing samples.

Table 1. Calculation of excess protons generated during the phase of severe stepwise normovolemic hemodilution with Voluven, Volulyte and Gelafundin whereby the exchanged volume of the last of nine steps of dilution was kept variable to reliably reach the final hematocrit of $10 \%$.

\begin{tabular}{lccc}
\hline $\begin{array}{l}\text { Volume replacement solution used for } \\
\text { hemodilution }\end{array}$ & $\begin{array}{c}\mathbf{C u r r e n t ~ H C O}_{\mathbf{3}}^{-} \\
\text {[mmol] }\end{array}$ & $\begin{array}{c}\text { Exchanged volume during } \\
\text { dilution [mI] }\end{array}$ & $\begin{array}{c}\text { Excess }^{+} \\
\text {[mmol] }\end{array}$ \\
\hline Voluven & 23 & 26 & $0.60 \pm 0.02$ \\
Volulyte & 26 & 26 & $0.68 \pm 0.02$ \\
Gelafundin & 23 & 25 & $0.58 \pm 0.04$ \\
\hline
\end{tabular}

Calculations were based on the values of six individual animals per group at three different points of time. Therefore, calculations using mean values above may differ. 


\section{Discussion}

During stepwise normovolemic hemodilution, bicarbonate is removed as a result of blood withdrawal and replacement of blood by non-bicarbonate-containing fluids. In the present experiments, the amount of bicarbonate removed that way approximated $0.6 \mathrm{mmol}$ (mean of the three experimental groups, Table 1). Since every molecule bicarbonate removed left behind one proton, the deficit of bicarbonate equaled the surplus of protons and thus, about $0.6 \mathrm{mmol}$ protons had been generated during the phase of hemodilution.

Generally, three different renal mechanisms exist to excrete or neutralize excess protons (Adam 1981, Rector 1973). First, excretion of free protons into urine. However, since the $\mathrm{pH}$ of urine is restricted between 4.5 and 7.5, only small amounts of protons can be excreted this way (Lim 2007, Rector 1973, Rose and Post 2001). In the present experiments, during hemodilution and the subsequent observation time, urinary $\mathrm{pH}$ decreased from approximately 7.3 to 6.3 (Teloh et al. 2013), indicating an additionally excreted amount of free protons of about $5 \cdot 10^{-6} \mathrm{mmol}$. Hence, the excretion of free protons is quantitatively negligible as compared to the amount of $0.6 \mathrm{mmol}$ protons formed during normovolemic hemodilution.

Second, removal of excess protons with the help of the phosphate buffer system. Attributable to the acidification of urine, $\mathrm{HPO}_{4}{ }^{2-}$ being present in the urine is further protonated to yield $\mathrm{H}_{2} \mathrm{PO}_{4}^{-}$. However, this buffer system is barely gradable due to limited available phosphate (Lim 2007, Pitts 1973, Rose and Post 2001). In our experiments, compared to the initial value, during and following hemodilution, the absolute amount of phosphate excreted is increased (Fig. 2), but these values are still around the reference of daily phosphate excretion in rats that amounts to approximately $0.35 \mathrm{mmol}$, i.e. 0.04 mmol within $3 \mathrm{~h}$ (Waynforth and Flecknell 1992). In total, excretion of protons via further phosphate protonation and increase in phosphate excretion may amount to approximately $0.03 \mathrm{mmol}$. Thus, as compared to a value in the order of $0.6 \mathrm{mmol}$ protons formed, this mechanism also does not constitute an adequate machinery to excrete the surplus of protons being generated during hemodilution.

Third, enhanced urinary ammonium excretion. Ammonium release from glutamine and hereinafter glutamate takes place in the kidney and is mediated by two enzymes, namely glutaminase I and glutamate dehydrogenase (Damian and Pitts 1970, Fahien and Strmecki 1969, Goldstein and Schooler 1967). In acidotic states, urinary ammonium excretion rises resulting from an interplay between renal glutamine degradation with a concomitant fall in glutamine concentration and increased ammonium excretion due to urinary acidification. In the liver, re-synthesis of glutamine occurs for disposal of waste nitrogen at the expense of ureagenesis. Since nitrogen excretion via ammonium does not utilize any bicarbonate, but nitrogen excretion via urea does, the saved bicarbonate can neutralize excess protons. Compared with the initial value, the absolute amount of ammonium in urine increased in all groups during experimental time (Fig. 1). According to the literature (Oliver and Bourke 1975), and also reference values of other in-house animals (unpublished data), animals are supposed to excrete approximately $0.42 \pm 0.01 \mathrm{mmol}$ ammonium within $24 \mathrm{~h}$ under physiological conditions. For the duration of the phase of dilution $(3 \mathrm{~h})$ and the subsequent observation phase $(2.5 \mathrm{~h})$, this amounts to $0.05 \mathrm{mmol}$ and $0.04 \mathrm{mmol}$, respectively. Therefore, the amount of ammonium excreted in the respective experimental phases ( $0.32 \mathrm{mmol}$ and $0.26 \mathrm{mmol}$, respectively, on average) is distinctly elevated as opposed to the aforementioned physiological baseline conditions. The sum of excreted ammonium during the phase of dilution and observation phase in each group was close to or even above $0.6 \mathrm{mmol}$. These values clearly show that the vast majority of protons produced during normovolemic hemodilution was compensated by urinary ammonium excretion. However, animals having received Gelafundin as volume replacement excreted in sum (phase of dilution and observation phase) approximately twice as much ammonium as animals of the Volulyte group, being the group that did excrete fewest of all (Gelafundin $0.88 \mathrm{mmol}$, Voluven $0.46 \mathrm{mmol}$ and Volulyte $0.40 \mathrm{mmol}$ ) suggesting a possible correlation of ammonium excretion with the colloid's chemical structure. Gelafundin comprises succinylated gelatin whose terminal carboxyl groups are deprotonated at physiological $\mathrm{pH}$, hence, carrying a negative charge. Due to the fact that succinylated gelatin is rapidly excreted via the urine (Teloh et al. 2013), it could have favored the excretion of cations like ammonium for electrochemical reasons. In contrast, hydroxyethyl starch, being the colloidal component in Voluven and Volulyte, is uncharged at physiological $\mathrm{pH}$. Based on the increased ammonium excretion in the Gelafundin group, one might even 
speculate that infused succinylated gelatin may lead to a disproportional ammonium excretion favoring alkalization. In addition to the colloid, the carrier solution's composition appears to be also of importance as regards the ability to excrete ammonium. Volulyte is the only solution of the three employed here whose carrier solution is plasma-adapted and therefore contains a metabolic anion in terms of acetate. Based on the theory of metabolizable anions (Zander 2009), metabolic acidosis should be antagonized by producing bicarbonate during their metabolization. Taking into account absolute values of ammonium excretion (Fig. 1), our data of the Volulyte group are in line with this theory. In sum (phase of dilution plus observation phase), urine contained only $0.40 \mathrm{mmol}$ ammonium (in contrast to $0.46 \mathrm{mmol}$ and $0.88 \mathrm{mmol}$ of the Voluven and Gelafundin group, respectively) but base excess values were simultaneously closest to neutrality of the three groups (Teloh et al. 2013), indicating, that the remaining surplus of protons is neutralized with the aid of bicarbonate originating from acetate metabolism.

In the past, a lot of experimental animal studies were conducted examining ammonium excretion as a response to acute metabolic acidosis (Bishop et al. 2010, Goldstein and Schooler 1967, Lee et al. 2009, Leonard and Orloff 1955, Oliver and Bourke 1975, Parry and Brosnan 1978, Veverbrants et al. 1977), showing, that urinary ammonia excretion is the primary component of the increase in net acid excretion (Bishop et al. 2010, Hamm and Simon 1987, Weiner and Verlander 2013). All of these studies more or less uniformly showed that metabolic acidosis enhanced urinary ammonium excretion within a few hours after acidosis induction. However, metabolic acidosis was always induced by administration of either hydrochloric acid or ammonium chloride. Therefore, these models were highly artificial compared to patients developing acute metabolic acidosis. In contrast, in the present study, normovolemic hemodilution was the employed model to establish an acidosis of dilutional origin which regularly develops in the clinical setting, especially in patients undergoing cardiac surgery with the need of an extracorporeal circulation (Teloh et al. 2017). Thus, we here confirmed that urinary ammonium excretion is also the primary component of net acid excretion in the context of normovolemic hemodilution and thus suffices to mitigate metabolic acidosis in terms of a compensatory mechanism. Additionally, differences in the need for urinary ammonium excretion became obvious due to the presence or absence of metabolizable anions in the colloidal volume replacement solution.

\section{Conflict of Interest}

There is no conflict of interest.

\section{References}

ADAM WR: Significance, mechanisms and control of renal ammoniagenesis. Aust N Z J Med 11: 6-10, 1981.

ASTRUP P, JORGENSEN K, ANDERSEN OS, ENGEL K: The acid-base metabolism. A new approach. Lancet 1: 1035-1039, 1960.

BISHOP JM, VERLANDER JW, LEE HW, NELSON RD, WEINER AJ, HANDLOGTEN ME, WEINER ID: Role of the Rhesus glycoprotein, Rh B glycoprotein, in renal ammonia excretion. Am J Physiol Renal Physiol 299: F1065-F1077, 2010.

DALY JA, ERTINGSHAUSEN G: Direct method for determining inorganic phosphate in serum with the "CentrifiChem". Clin Chem 18: 263-265, 1972.

DAMIAN AC, PITTS RF: Rates of glutaminase I and glutamine synthetase reactions in rat kidney in vivo. $A m J$ Physiol 218: 1249-1255, 1970.

FAHIEN LA, STRMECKI M: Studies on gluconeogenic mitochondrial enzymes. II. The conversion of glutamate to alpha-ketoglutarate by bovine liver mitochondrial glutamate dehydrogenase and glutamate-oxaloacetate transaminase. Arch Biochem Biophys 130: 456-467, 1969.

GOLDSTEIN L, SCHOOLER JM: Regulation of ammonia production in the rat kidney. Adv Enzyme Regul 5: 71-86, 1967.

GUNNERSON KJ, SAUL M, HE S, KELLUM JA: Lactate versus non-lactate metabolic acidosis: a retrospective outcome evaluation of critically ill patients. Crit Care 10: R22, 2006.

HAMM LL, SIMON EE: Roles and mechanisms of urinary buffer excretion. Am J Physiol 253: F595-F605, 1987. 
KRAUT JA, MADIAS NE: Treatment of acute metabolic acidosis: a pathophysiologic approach. Nat Rev Nephrol 8: 589-601, 2012.

LEE HB, BLAUFOX MD: Blood volume in the rat. $J$ Nucl Med 26: 72-76, 1985.

LEE HW, VERLANDER JW, BISHOP JM, IGARASHI P, HANDLOGTEN ME, WEINER ID: Collecting ductspecific $\mathrm{Rh} \mathrm{C}$ glycoprotein deletion alters basal and acidosis-stimulated renal ammonia excretion. Am J Physiol Renal Physiol 296: F1364-F1375, 2009.

LEONARD E, ORLOFF J: Regulation of ammonia excretion in the rat. Am J Physiol 182: 131-138, 1955.

LIM S: Metabolic acidosis. Acta Med Indones 39: 145-150, 2007.

OLIVA PB: Lactic acidosis. Am J Med 48: 209-225, 1970.

OLIVER J, BOURKE E: Adaptations in urea ammonium excretion in metabolic acidosis in the rat: a reinterpretation. Clin Sci Mol Med Suppl 48: 515-520, 1975.

PARRY DM, BROSNAN JT: Glutamine metabolism in the kidney during induction of, and recovery from, metabolic acidosis in the rat. Biochem J 174: 387-396, 1978.

PITTS RF: Production and excretion of ammoina in relation to acid-base regulation. In: Renal Physiology. J ORLOFF, RW BERLINER (eds), American Physiological Society, Washington, 1973, pp 455-496.

RECTOR FC JR: Acidification of the urine. In: Renal Physiology. J ORLOFF, RW BERLINER (eds), American Physiological Society, Washington, 1973, pp 431-454.

ROSE BD, POST TW: Regulation of acid-base balance. In: Clinical Physiology of Acid-Base and Electrolyte Disorders. BD ROSE, TW POST (eds), McGraw-Hill, New York, 2001, pp 325-371.

TELOH JK, FERENZ KB, PETRAT F, MAYER C, DE GROOT H: Acid-base and electrolyte status during normovolemic hemodilution with succinylated gelatin or HES-containing volume replacement solutions in rats. PLoS One 8: e72848, 2013.

TELOH JK, DOHLE D-S, SÖNMEZ S, TSAGAKIS K, VERHAEGH R, PETERSEN M, JAKOB H, DE GROOT H: Transient dilutional acidosis but no lactic acidosis upon cardiopulmonary bypass in patients undergoing coronary artery bypass grafting. Arch Med Sci 13: 585-590, 2017.

VEVERBRANTS E, CHAZAN JA, GARELLA S: Ammonium excretion in response to acid loads: effect of metabolic alkalosis and cation depletion. $J$ Lab Clin Med 89: 1251-1261, 1977.

WATERS JH, MILLER LR, CLACK S, KIM JV: Cause of metabolic acidosis in prolonged surgery. Crit Care Med 27: 2142-2146, 1999.

WEINER ID, VERLANDER JW: Renal ammonia metabolism and transport. Compr Physiol 3: 201-220, 2013. 